\title{
THE ORIGIN OF URINARY CREATINE IN PROGRESSIVE MUSCULAR DYSTROPHY ${ }^{1}$
}

\author{
BY JEAN D. BENEDICT,2 HELEN J. KALINSKY, LOUIS A. SCARRONE, ARTHUR \\ R. WERTHEIM, AND DEWITT STETTEN, JR.2 \\ (From the Division of Nutrition and Physiology, The Public Health Research Institute of the \\ City of New York, Inc., and Columbia Research Service, Goldwater \\ Memorial Hospital)
}

(Submitted for publication March 22, 1954; accepted August 27, 1954)

In an earlier communication (1) an experiment was described in which the isotopic compositions of urinary creatine and creatinine excreted by a patient with progressive muscular dystrophy were studied after a feeding of glycine- $\mathrm{N}^{15}$. It was found, that in the first few days urinary creatine was much more enriched with $\mathrm{N}^{15}$ than was urinary creatinine, and that the isotope concentration in the creatine fell far more rapidly than did that in the creatinine. These results were taken to mean that the creatinuria, in this patient, was not a consequence of leakage of creatine out of skeletal muscle but rather represented newly formed creatine, synthesized in liver, which had failed to gain entry into muscle.

The present study represents an extension of these observations on two additional patients. Essentially the same technique as previously described was utilized. In view of the fact that the "sarcosine-nitrogen" of creatine derives from glycine nitrogen in liver, as does the nitrogen of hippuric acid, it was considered of interest to compare the abundance of $\mathrm{N}^{15}$ in simultaneously excreted creatine and hippurate after feeding glycine- $\mathrm{N}^{15}$. To facilitate hippurate isolation from urine, small amounts of sodium benzoate were administered from time to time to each subject.

\section{EXPERIMENTAL}

\section{Clinical histories}

Subject T. K., a 31-year-old white male, has exhibited evidences of muscle dysfunction since the age of 4 and was diagnosed as having progressive muscular dystrophy

1 This investigation was supported in part by a research grant (A-324) from The National Institute of Arthritis and Metabolic Diseases, National Institutes of Health, Public Health Service.

2 Present Address:

National Institute of Arthritis and Metabolic Diseases, National Institutes of Health, Bethesda 14, Maryland. at the age of 13 . He has been unable to walk for the past 15 years. Marked wasting of the muscles of the shoulders, upper arms, spine, and thighs is apparent with some pseudohypertrophy of forearms, calves, and gluteal muscles. There was no evidence of disease of the nervous system.

Subject S. I. is a 28-year-old white male who has suffered from progressive muscular weakness since the age of 8 . He has been unable to walk since the age of 16. Severe atrophy is noted in muscles of neck, upper arms, forearms, glutei, and thighs. He was considered a classical example of advanced progressive muscular dystrophy.

\section{Experimental procedure}

The subjects were maintained on a creatine-free, purine-poor diet throughout the experimental period. After four or five days, glycine- $\mathrm{N}^{15}$ was fed at 9 A.M. The dosage, as in the earlier experiments, was $100 \mathrm{mg}$. glycine per kilogram of body weight $\left(\mathrm{N}^{15}=60.2\right.$ atom per cent excess). Each patient was continued on the diet for 14 days, and total urine was collected in 24-hour samples.

During this period, subject $T$. $K$. excreted an average of $320 \mathrm{mg}$. of creatine and $114 \mathrm{mg}$. of creatinine daily. Subject S. I. showed an average daily excretion of 685 mg. of creatine and $189 \mathrm{mg}$. of creatinine.

In the experiment on $T$. $K$. sodium benzoate, $450 \mathrm{mg}$., was injected intravenously immediately after breakfast on the 1st, 2nd, 4th and 8th day. Subject S. I. was given intravenous sodium benzoate two hours before breakfast on days $1,2,4$ and 8 . On day 9 he received sodium benzoate after breakfast to check the influence of a meal on the excretion of $\mathrm{N}^{15}$. No essential difference was noted. The urine excreted during the two hours following each injection of sodium benzoate was separately collected, acidified to $\mathrm{pH} 3$ with $\mathrm{HCl}$ and continuously extracted with ether for 12 hours. The ethereal extract was evaporated and the residue, after treatment with activated charcoal, was recrystallized from hot water. The melting points of the hippuric acid ranged between 187 to $189^{\circ}$.

Synthesis, isolations and degradations

Glycine- $\mathrm{N}^{15}$ was prepared essentially as described by Schoenheimer and Ratner (2) as modified by Muller and Bauer (3).

Each 24-hour urine sample was acidified with $\mathrm{HCl}$ to pH 3 or 4 and was then mechanically stirred with $30 \mathrm{gm}$. 
of Lloyd's reagent for three hours. The supernatant fluid, after centrifugation; was set aside. The solid phase was washed once with $0.1 \mathrm{~N} \mathrm{HCl}$. A preliminary study had revealed that essentially quantitative separation of creatine from creatinine was effected by this procedure, the creatinine being absorbed and the creatine remaining in the supernatant fluid. Creatinine was recovered from Lloyd's reagent by repeated elution with saturated aqueous $\mathrm{Ba}(\mathrm{OH})_{2}$ solution until the eluate failed to give a Jaffe reaction. After treatment with $\mathrm{CO}_{2}$ and removal of $\mathrm{BaCO}_{3}$, the solution was evaporated under reduced pressure to about $10 \mathrm{ml}$., acidified to Congo Red paper with acetic acid and filtered if necessary. Creatinine was determined colorimetrically and one equivalent each of picric acid and potassium picrate were added in aqueous solution. The precipitate which formed over night was collected and recrystallized, as suggested by Bloch and Schoenheimer (4), from 0.12 per cent aqueous picric acid.

$$
\begin{aligned}
& \mathrm{C}_{4} \mathrm{H}_{7} \mathrm{ON}_{8}+\mathrm{C}_{6} \mathrm{H}_{8} \mathrm{O}_{7} \mathrm{~N}_{8}+\mathrm{KC}_{6} \mathrm{H}_{2} \mathrm{O}_{3} \mathrm{~N}_{7} \\
& \text { Calculated, creatinine } 18.3 \\
& \text { Found, creatinine } 18.5
\end{aligned}
$$

A weighed sample of potassium creatinine picrate was suspended in aqueous acid and exhaustively extracted with ether. A Kjeldahl nitrogen determination was conducted upon the colorless aqueous phase.

$$
\begin{aligned}
& \mathrm{C}_{6} \mathrm{H}_{7} \mathrm{ON}_{8}+\mathrm{C}_{6} \mathrm{H}_{3} \mathrm{O}_{7} \mathrm{~N}_{2}+\mathrm{KC}_{6} \mathrm{H}_{2} \mathrm{O}_{3} \mathrm{~N}_{7} \\
& \text { Calculated, creatinine } \mathrm{N} 6.9 \\
& \text { Found, creatinine N } 6.9
\end{aligned}
$$

The supernatant solution from the treatment with Lloyd's reagent was treated with $42 \mathrm{ml}$. of conc. $\mathrm{HCl}$ per liter and the mixture was autoclaved at $15 \mathrm{lb}$. pressure for one hour, to convert creatine into creatinine. The latter product was then isolated as its potassium picrate double salt by the same procedure as is described above.

A trial separation was carried out by the foregoing procedures upon a synthetic mixture of creatinine $\left(\mathrm{N}^{13}=\right.$ 0.073 atom per cent excess) and creatine $\left(\mathrm{N}^{16}=0\right.$ atom per cent excess). Analyses of the separated components revealed no detectable isotopic enrichment in the creatine component, whereas the creatinine component contained 0.073 atom per cent excess.

Approximately $20 \mathrm{mg}$. of each sample of double salt were suspended in $0.1 \mathrm{~N} \mathrm{HCl}$ and continuously extracted with boiling ether. Ether extraction was continued for at least one-half hour after the disappearance of yellow color from the aqueous phase. The colorless aqueous solution of creatinine hydrochloride was then digested by the $\mathrm{Kjeldahl}$ procedure in order to prepare the samples for mass spectrometric analyses of $\mathrm{N}$.

The distribution of isotope in creatine was studied by the alkaline degradation of creatinine samples derived from urinary creatine. About $30 \mathrm{mg}$. of picrate double salt were heated under reflux in about $70 \mathrm{ml}$. of saturated aqueous $\mathrm{Ba}(\mathrm{OH})_{2}$ solution. A continuous stream of $\mathrm{N}_{2}$ swept the ammonia, formed from the amidine group of creatinine, into a receiver containing $5 \mathrm{ml}$. of 4 per cent boric acid solution (5). $\mathrm{N}^{15}$ abundance was determined in this ammonia, and from this value and the corresponding analysis of the creatine, the abundance of $\mathrm{N}^{15}$ in the sarcosine moiety was calculated from the equation:

where

$$
\mathrm{N}_{\mathrm{s}}=3 \mathrm{~N}_{\mathrm{o}}-2 \mathrm{~N}_{\Delta}
$$

$\mathrm{N}_{\mathrm{s}}=$ atom per cent excess $\mathrm{N}^{15}$ in sarcosine moiety

$\mathrm{N}_{c}=$ atom per cent excess $\mathrm{N}^{1 s}$ in creatine

$\mathrm{N}_{\Delta}=$ atom per cent excess $\mathrm{N}^{15}$ in ammonia liberated.

Analyses for creatine and creatinine were conducted by a modified Folin-Wu (6) method.

\section{RESULTS AND DISCUSSION}

The present experiments were predicated upon the assumption that if creatine and creatinine of the urine both arose from the same muscle creatine, they would at all times exhibit the same isotope enrichment. In our earlier study, following the feeding of glycine- $\mathrm{N}^{15}$ to a patient with progressive muscular dystrophy, this was found not to be the case. The results obtained in the present study (Figures 1 and 2) are in good qualitative agreement with the values previously published. Initially, far higher concentrations of $\mathrm{N}^{15}$ are found in urinary creatine than in urinary creatinine. The isotope concentration in creatine falls rapidly and by the 8 th or 10 th day is not sensibly different from that in the creatinine.

Creatinine is generally believed to arise in skeletal muscle from muscle creatine or phosphocrea-

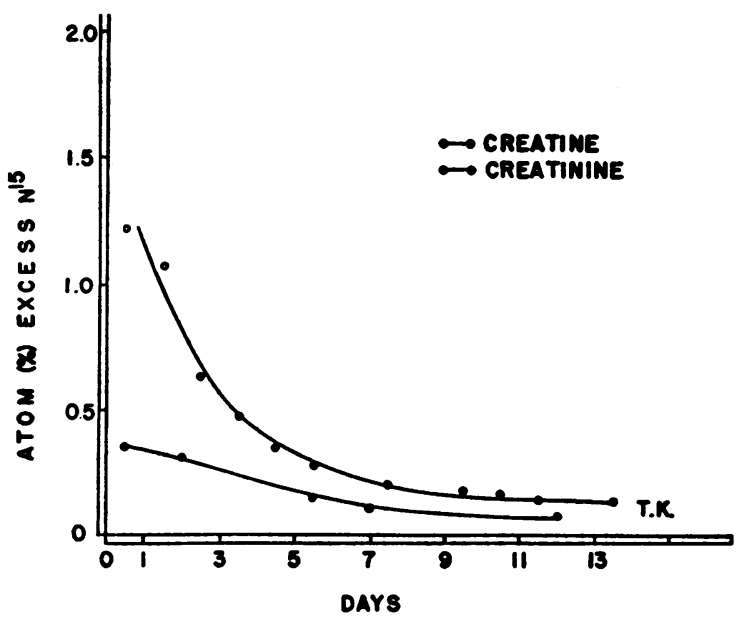

Fig. 1. Atom Per Cent Excess N ${ }^{2}$ in Urinary Creatine and Creatinine in a Patient with ProGRESSIVE MUSCULAR DYSTROPHY AFTER INGESTION OF GLYCINE-N" 


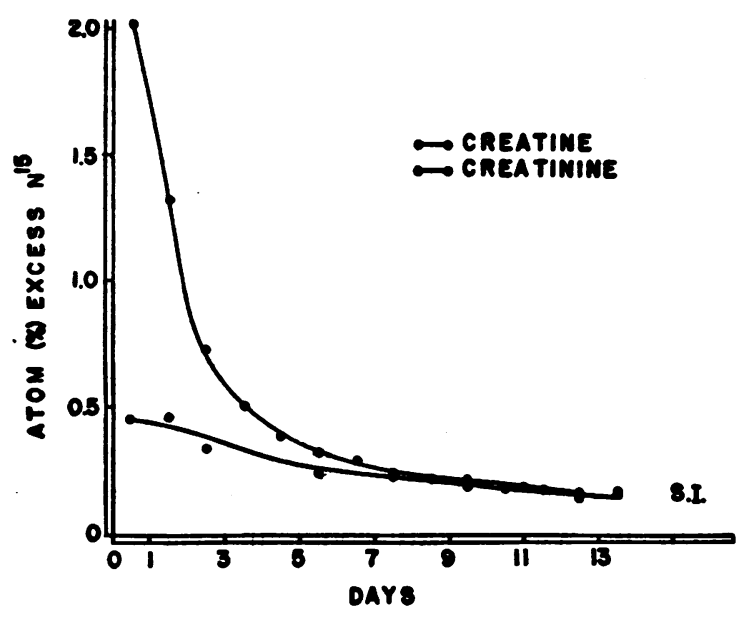

Fig. 2. Atom Per Cent Excess $N^{\text {Is }}$ in Urinary Creatine and Creatinine in a Patient with Progressive Muscular Dystrophy after Ingestion OF GLYCINE-N"

tine (7). Isotope studies in animals $(4,8)$ have revealed that after suitable labeling procedures, the label is present in urinary creatinine at the same concentration as occurs simultaneously in muscle creatine, and this leads to the inference that muscle creatine is the unique precursor of urinary creatinine.

The present findings are taken to confirm our earlier conclusion that the urinary creatine in this disease did not leak out of muscle. From its initial high isotopic enrichment and from the subsequent rapid decline in abundance of $\mathrm{N}^{15}$ it is concluded that the creatine of the urine, in this disease, is freshly synthesized creatine which has never been mixed with the large stores of creatine contained in skeletal muscle. In man there is reason to believe that most or all creatine synthesis occurs in the liver (9) and it is, therefore, suggested that in progressive muscular dystrophy the creatinuria is a consequence of an imbalance between normal hepatic creatine synthesis, and markedly diminished creatine uptake by muscle. This view is in accord with that expressed by Hoagland, Gilder, and Shank (10).

The isotope concentrations observed in urinary creatinine in these two patients as well as in the patient studied previously, are higher than the corresponding values obtained from normal subjects (1). This suggests that, despite the great diminution in functional muscle mass, such muscle as survives is still very competent to convert creatine into creatinine. In fact the fraction of muscle creatine converted to creatinine daily is, if anything, greater than normal, although the absolute amount of creatinine formed is obviously less than normal.

It has been clearly established that the synthesis of creatine in animals involves the amidination of glycine at the expense of arginine followed by methylation of glycocyamine, at the expense of methionine. Glycine has been shown to be a specific biological donor of nitrogen to the sarcosine fragment obtained by alkaline degradation of creatine (11). Since this utilization of glycine in man is supposed to be largely a liver function and since hippurate formation is employed clinically as a measure of liver function, it was considered of interest to compare, after glycine- $\mathrm{N}^{15}$ ingestion, the concentrations of $\mathrm{N}^{15}$ in simultaneously excreted creatine and hippurate samples. The creatine values have been corrected for isotope contained in the amidine portion of the molecule (Tables I and II) and the results have been plotted on semilogarithmic coordinates, together with the hippurate data, in Figures 3 and 4.

Several points of interest emerge from these curves. In the first place, at all points in time in both subjects, there is a higher concentration of isotope in the sarcosine moiety of creatine than in the hippurate. Furthermore, the two curves, in each experiment, are roughly parallel on the coordinates employed, and indicate a fairly uniform ratio of isotope concentrations in the two products. The concentration of isotope in the sarcosine is

TABLE I

Abundance and distribution of $N^{15}$ in urinary creatine -Subject T. $K$.* $^{*}$

\begin{tabular}{rccc}
\hline \hline Day & Creatine & Amidine & Sarcosine \\
\hline 1.5 & 1.070 & 0.558 & 2.096 \\
2.5 & 0.634 & 0.348 & 1.206 \\
3.5 & 0.475 & 0.251 & 0.921 \\
4.5 & 0.352 & 0.180 & 0.695 \\
5.5 & 0.281 & 0.142 & 0.558 \\
7.5 & 0.200 & 0.102 & 0.397 \\
9.5 & 0.176 & 0.088 & 0.352 \\
10.5 & 0.170 & 0.089 & 0.333 \\
11.5 & 0.154 & 0.076 & 0.308 \\
13.5 & 0.141 & 0.066 & 0.291 \\
\hline
\end{tabular}

* From the concentrations of $\mathrm{N}^{16}$ in creatine samples and in the amidine nitrogen liberated from these samples, the concentrations of $\mathrm{N}^{16}$ in the sarcosine moiety have been calculated. 
TABLE II

Abundance and distribution of $N^{16}$ in urinary creatine -Subject S. I.*

\begin{tabular}{rccc}
\hline \hline Day & Creatine & Amidine & Sarcosine \\
\hline 0.5 & 2.021 & 0.452 & 5.159 \\
1.5 & 1.322 & 0.443 & 3.080 \\
2.5 & 0.730 & 0.179 & 1.833 \\
4.5 & 0.390 & 0.133 & 0.905 \\
5.5 & 0326 & 0.102 & 0.774 \\
7.5 & 0.293 & 0.110 & 0.529 \\
8.5 & 0.250 & 0.081 & 0.482 \\
9.5 & 0.218 & 0.075 & 0.502 \\
10.5 & 0.194 & 0.062 & 0.459 \\
12.5 & 0.167 & 0.079 & 0.345 \\
13.5 & 0.163 & 0.075 & 0.338 \\
\hline
\end{tabular}

* From the concentrations of $\mathrm{N}^{16}$ in creatine samples and in the amidine nitrogen liberated from these samples, the concentrations of $\mathrm{N}^{15}$ in the sarcosine moiety have been calculated.

two to three times as high as in the hippuric acid. An additional point worthy of note is that the decline in isotope concentration is in no case a logarithmic function of time, indicating that this is not a simple first order process.

It had been anticipated that insofar as creatine synthesis and hippurate synthesis both tapped the glycine reserve of the liver, both products might show the same isotope concentrations in the present experiment. Two explanations may be invoked to account for the present finding of consistently greater enrichment of the sarcosine portion of creatine. The first of these relates to the possibility that between the moment of removal of a glycine molecule and the final excretion of the

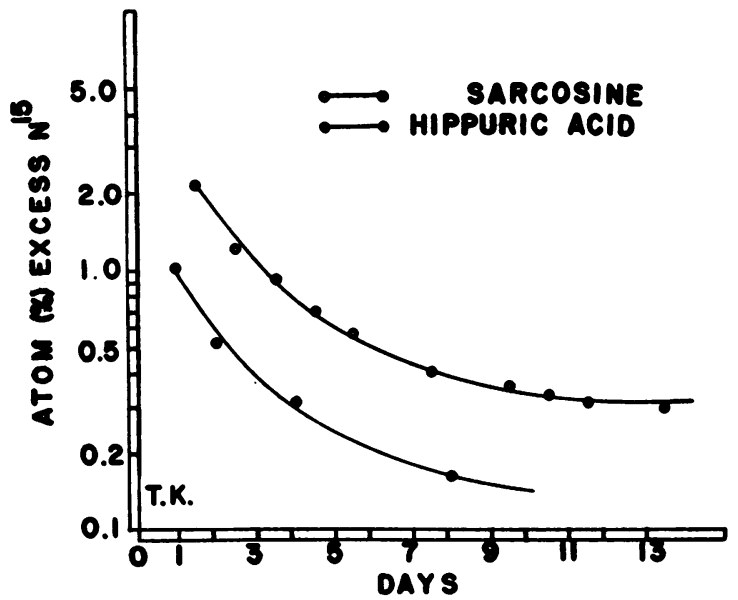

Fig. 3. Atom Per Cent Excess $\mathrm{N}^{\text {w }}$ in Hippurate and in Sarcosine Moiety of Creatine creatine molecule formed from it, a significant lapse of time intervenes. The creatine excreted at any time would then represent liver glycine at some earlier time, and since throughout most of the experimental period the isotope enrichment in liver glycine must be falling, the creatine excreted would be richer in isotope than the glycine, present in the liver, available for hippurate synthesis at that time. This explanation of itself appears to be incomplete. Such a time lag, did it occur, would be expected to be constant during the experiment. A comparison of the points in time on the two curves at which both compounds are equally labeled (Figure 3 or 4 ) reveals an initial time interval of about two days, but this becomes far longer as the experiment progresses. The failure

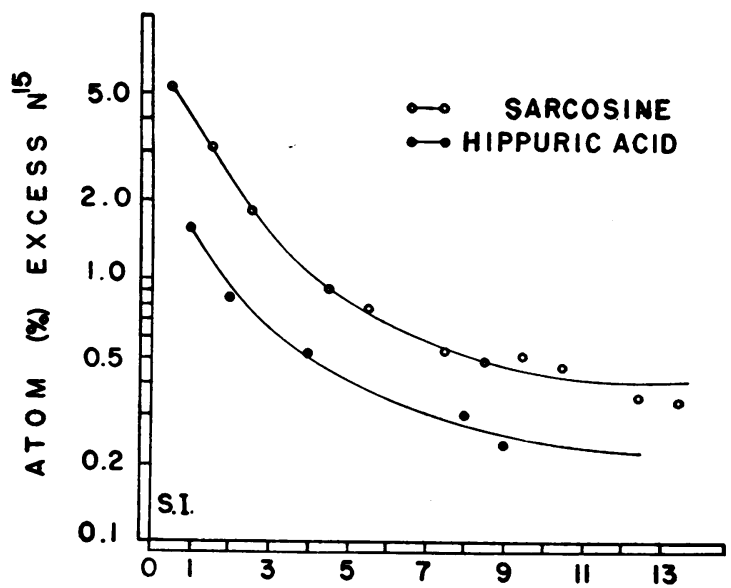

Fig. 4. Atom Per Cent Excess $\mathrm{N}^{15}$ In Hippurate and in Sarcosine Moiety of Creatine

to detect, in either experiment the ascending limb of the curve of $\mathrm{N}^{15}$ in sarcosine also argues against the occurrence of any significant time lag in creatine synthesis or in creatine excretion.

An alternative explanation is that there are in the liver at least two pools of glycine nitrogen which are not perfectly mixed with each other. Various mechanisms may be envisioned to account for the greater dilution of $\mathrm{N}^{15}$ in one pool than in the other, but at present information is insufficient to warrant speculation. The suggestion inherent in these results that isotope may be far from uniform in its distribution in one compound in one organ interjects a note of caution into interpretations of results of isotope experiments 
in which homogeneous distribution of isotope must be assumed.

The possibility that the abrupt drain upon the supply of glycine in the liver imposed by the injection of sodium benzoate might of itself reduce the abundance of $\mathrm{N}^{15}$ in glycine has been considered. With this in mind we have kept the dosage of benzoate relatively small. However, even if this small additional drain of glycine did result. in an abrupt decline in $\mathrm{N}^{15}$ concentration in that portion of glycine in liver used for hippurate formation, this change was not reflected in the sarcosine data. Consequently the conclusion that these two glycine derivatives tap discrete and imperfectly mixed reservoirs appears inescapable.

\section{SUM MARY}

Two patients with severe progressive muscular dystrophy have been fed glycine- $\mathrm{N}^{15}$ while on a creatine-free diet. The concentrations of $\mathrm{N}^{15}$ in urinary creatine and creatinine have been measured and compared. Initially far higher concentrations of isotope were found in creatine than in creatinine, and the decline in isotope abundance with time was more rapid in creatine than in creatinine.

These findings indicate that urinary creatine. in this disease, does not originate in muscle but represents freshly synthesized creatine which has been denied access to muscle.

A comparison of $\mathrm{N}^{15}$ concentrations in simultaneously excreted creatine and hippuric acid samples revealed that, at all points in time studied, the concentration of isotope in the sarcosine moiety of creatine exceeded that in hippuric acid by a factor of two- to three-fold. This finding has been taken as evidence of inhomogeneity in the glycine of liver available for synthetic processes.

\section{ACKNOWLEDGMENT}

The authors are indebted to Miss Vida Klybas and Mr. Frank J. Rennie for the performance of isotope aralyses.

\section{REFERENCES}

1. Benedict, J. D., Roche, M., Yu, T. F., Bien, E. J., Gutman, A. B., and Stetten, D., Jr., Incorporation of glycine nitrogen into uric acid in normal and gouty man. Metabolism 1952, 1, 3.

2. Schoenheimer, R., and Ratner, S., Studies in protein metabolism. III. Synthesis of amino acids containing isotopic nitrogen. J. Biol. Chem., 1939, 127, 301.

3. Muller, A. F., and Bauer, W., Uric acid production in normal and gouty subjects determined by $\mathrm{N}^{15}$ labeled glycine. Proc. Soc. Exper. Biol. \& Med., 1953, 82, 47.

4. Bloch, K., and Schoenheimer, R., Studies in protein metabolism. XI. The metabolic relation of creatine and creatinine studied with isotopic nitrogen. J. Biol. Chem., 1939, 131, 111.

5. Gaebler, O. H., The decomposition of creatinine with baryta. J. Biol. Chem., 1926, 69, 613.

6. Kennedy, T. J., Hilton, J. G., and Berliner, R. W., Comparison of inulin and creatinine clearance in the normal dog. Am. J. Physiol., 1952, 171, 164.

7. Borsook, H., and Dubnoff, J. W., The hydrolysis of phosphocreatine and the origin of urinary creatinine. J. Biol. Chem., 1947, 168, 493.

8. DuVigneaud, V., Cohn, M., Chandler, J. P., Schenck, J. R., and Simmonds, S., The utilization of the methyl group of methionine in the biological synthesis of choline and creatine. J. Biol. Chem., 1941, 140, 625.

9. Sandberg, A. A., Hecht, H. H., and Tyler, F. H., Studies in disorders of muscle. $\mathrm{X}$. The site of creatine synthesis in the human. Metabolism, 1953, $2,22$.

10. Hoagland, C. L., Gilder, H., and Shank, R. E., The synthesis, storage, and excretion of creatine, creatinine, and glycocyamine in progressive muscular dystrophy and the effects of certain hormones on these processes. J. Exper. Med., 1945, 81, 423.

11. Bloch, K., and Schcenheimer, R., The biological precursors of creatine. J. Biol. Chem., 1941, 138, 167. 